\title{
New Strategy to Optimize the Performance of Epidemic Routing Protocol
}

\author{
Mohamed ababou \\ INPT \\ Madinat AL Irfane - \\ Rabat-Marocco
}

\author{
Rachid Elkouch \\ INPT \\ Madinat AL Irfane - \\ Rabat-Marocco
}

\author{
Mostata Bellafkih \\ INPT \\ Madinat AL Irfane - \\ Rabat -Marocco
}

\author{
Nabil Ababou \\ University Mohamed 1 \\ FSO, Oujda, Morocco
}

\begin{abstract}
The delay tolerant networks (DTN, Delay Tolerant Networks) are networks where connectivity is intermittent because of the difficulties encountered in the environment such as climate, mobility, the breakdowns of energy, etc. To achieve a good delivery of data despite all these challenges, new network architecture was developed. This one consists on the addition of a supplementary layer in the OSI protocol stack, above the transport layer. This new policy is called: the bundle protocol. Its main role is to store data until an opportunity of transmission appears by using the Store and Forward technique and the concept of custody transfer. Routing protocols in this type of network do not try to find the shortest path as it is the case in the conventional protocols, but they focus on the increase in data delivery. The techniques used for this purpose can be divided into two large families. Some protocols adopt the approach of replication, which involves the duplication of messages in the network by providing multiple copies in order to increase the likelihood of transmission. Others, are based on knowledge, that is to gather information about the network status and manage shipments efficiently. In DTN routing protocol forwarding decision and buffer management strategy are important to improve the chance of message delivery. In this paper, the new strategy proposed optimizes Epidemic routing protocol; it consists on using the path of ACKs messages and the path of all messages that have reached their destination to resolve the disadvantages of Epidemic routing protocol with the FIFO method. According to the simulation, the proposed method shows better delivery rate, better delivery probability and lower communication overhead compared to FIFO strategy and it is suitable for Epidemic.
\end{abstract}

\section{General Terms}

Algorithm, Routing, Epidemic router.

\section{Keywords}

Store and forward, forwarding strategies, routing, DTN, Queue modes.

\section{INTRODUCTION}

Delay Tolerant Networks (DTN) is a concept initially created for interplanetary networks [1]. However, Crowcroft, Yoneki, Hui, and Henderson said that it also receives a large success for irregularly connected networks and principally for opportunistic networks [2].

The aim of a DTN is to supply reliable communication in environments involving frequent disruption of network connections and extremely long delays in transmission.

In these networks, a node can send data to another one if they are within the transmission range of each other. Due to the active character of these networks, there is no assurance that a direct connected path from a given source to a given destination exists at any time.

As a result, and as it was confirmed by Vahdat and Becker, DTN routing enables communication in reduced environments through replication of message from source node to relay nodes and delivery of the data to the destination by one of the nodes such as 'Epidemic' [3] and 'Spray and Wait' [4] that have been proposed to increase the message delivery ratio over such intermittently connected networks.

The performance evaluation of such protocols in terms of message delivery ratio and probability of deliverance is a difficult task due to the complexity to drive mobile network simulations. Several efforts have been done in order to evaluate the performance of routing schemes with simulations. Today, as it was approved by Helsinki University of Technology, especially the Networking Laboratory, The ONE simulator became a reference tool in this area [5].

Thus, in DTN, each node uses a store-carry-forward technique to send a message [2-6]. When a node receives a message, it stores a message and carries that message when it moves. Then, it forwards the message to other relay nodes or a destination node. Forwarding a message based on hop-by-hop routing decision is more practical than the one that is based on finding a fully connected end-to-end path.

The key problem is hence, how to make effective forwarding decisions, to ensure that the messages are carried by relays with the best chance to contact their destinations.

In this paper the proposed forwarding strategy Supp-Tran, provides a quality of service and optimizes the performance of epidemic routing protocol in terms of delivery probability, overhead ratio and Hop count average. The remaining paper is designed as follows: Section 2, elaborates existing forwarding strategies. Section 3, is about routing protocol in DTN networks. Section 4, deals with used mobility models. Section 5 , is mainly about performance metrics. Section 6 presents the approach. Section 7, deals with the Simulation and its results followed by a conclusion in section 8 .

\section{FORWARDING STRATEGIES}

\subsection{GRTR}

When two nodes A and B meet, the strategy is to calculate $\mathrm{P}(\mathrm{A}, \mathrm{D})$ and $\mathrm{P}(\mathrm{B}, \mathrm{D})$ which denotes respectively the delivery predictability [7] that a node $A$ and $B$ have for a destination $\mathrm{D}$ then the message must be carried by a node that has a higher delivery predictability for the destination of the message

\subsection{MOFO}

This strategy records the number of times that message has been sent and classifies it according to a descending order, the 
more the number of the sent message is low, the more the message has a chance of being transmitted [8]. If the buffer is full the strategy deletes messages that have been sent many times. Messages that were sent several times are not sent and the ones that have not been sent or transmitted many times are sent because this increases their chances of reaching their destination.

\subsection{First in First out (FIFO)}

In FIFO queue mode all messages are organized according to their arrival time and the message which has the oldest arrival time will be sent first. If the buffer is full, the message that entered first into the queue is the first message to be dropped.

\subsection{Random Queue Mode (RND)}

The message is randomly selected for the transmission to be dropped or sent.

\section{PROTOCOL UNDER OBSERVATION}

\subsection{Epidemic Routing Protocol}

Epidemic routing [3] has been proposed as an approach for DTN networks in which there may not be an end to end path from source to destination. It adopts a store-carry-forward mechanism when a node receives a message it buffers and carries it as it moves. Then, it sends the message to new nodes that it encounters. This is similar to infectious diseases. The destination receives the message when it first meets a node that carries it. Epidemic routing is able to attain minimum delivery delay at the cost of increased use of resources such as buffer space, bandwidth, and transmission power.

\section{ABOUT MOBILITY MODELS USED}

\subsection{SPMBM}

SPMBM (Shortest Path Map Based Movement Model) [5] is a more realistic model it manages the movement of nodes in the simulation map scenario. It will provide destination coordinates, speeds, wait times, and uses Dijkstra's algorithm to find the shortest path to the destination. It places the nodes in random places but selects a certain destination in the map for all nodes.

\subsection{Route Based Models}

In this model, MBM [5], nodes travel randomly but always follow the determined paths defined by the map of simulation scenario which is effectively used for simulation of movement of nodes as in the case of buses and trams, determines stops during which nodes wait for a while before continuing their paths along the shortest way.

\section{THE PERFOMANCE METRICES MEASURED}

As it was stated in many researches, in order to compare routing strategies, some parameters need to be defined to evaluate their performance. The number of the selected metrics depends on several factors.

\subsection{Overhead Ratio}

Overhead ratio [5] can be defined as the subtraction of the bundle carried $(\mathrm{BC})$ and the bundles delivered (BD) over bundles carried ((BC-BD)/BC). The overhead ratio reflects how many unnecessary messages are relayed to deliver one message. It reflects transmission cost in a network. The more the value of overhead is low the more the strategy used is efficient; this leads to a minimization of consumption of the network resources.

\subsection{Delivery Probability}

It is the ratio of the message delivered over message relayed. High probability means that more messages are delivered to the destination.

\subsection{Hop Count Average}

It is the mean of the number of hops which participate to relayed message from its source to its destination successfully; higher values mean that the message has consumed many network resources before reaching its destination

\section{THE STRATEGY SUP-TRAN}

All nodes are mobile with a low density. There is no connection from end to end, and the movement of nodes affects the delivery of the message. When two nodes meet each other, they exchange the messages that they carry. See Fig1. below of an example of a scenario which shows given the location of nodes changes exponentially with the time.

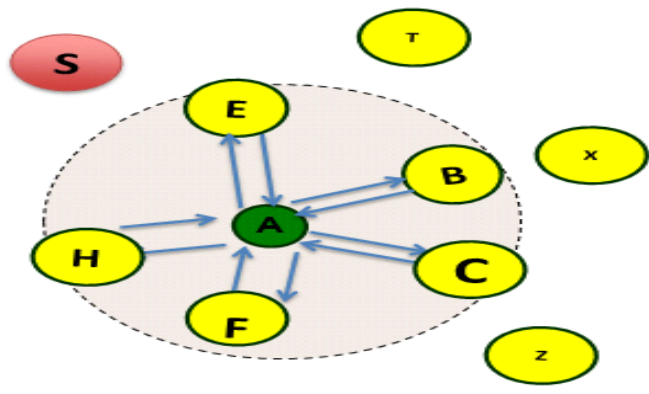

Fig1. Example of a scenario

\subsection{Cleaning the Buffer}

\subsubsection{Phase gathering information.}

When two nodes A and B meet, they exchange their two lists namely the list of neighbors and the list of unnecessary messages:

-List of neighbors

$$
\mathrm{VA}=\{\mathrm{VAi}=\mathrm{Ai}, \mathrm{i}=1, \ldots, \mathrm{n}\}
$$

with n: number of neighbors of A.

$$
\mathrm{VB}=\{\mathrm{VBi}=\mathrm{Bi}, \mathrm{i}=1, \ldots, \mathrm{m}\}
$$

with m: number of neighbors of $\mathrm{B}$.

$\mathrm{VAi}$ and $\mathrm{VBi}$ are neighbors respectively of $\mathrm{A}$ and $\mathrm{B}$.

- List of unnecessary messages.

ListeMAN: List of messages that a node must not accept, it contains messages that have already arrived at their destinations.

ListeMAN (VAi): The list of messages that the neighbor (Ai) of node (A) shall not accept.

$$
\text { ListeMAN }(\mathrm{A})=\{\operatorname{ListeMAN}(\mathrm{VAi}), \mathrm{i}=1, \ldots, \mathrm{n}\}
$$

Where $\mathrm{n}$ : the number of neighbors of A.

$$
\text { ListeMAN }(B)=\{\text { ListeMAN }(\mathrm{VBi}), \mathrm{i}=1 \ldots \mathrm{m}\}
$$

Where m: the number of neighbors of $B$.

\subsubsection{Creation of ListeMAN (AB)}

It consists on the creation of a new list that contains the messages to remove, by the union of the two previous lists that is to say those that emanate from multiple nodes - in order to increase the removal rate of unnecessary messages 
circulating in the network even if a copy has already arrived successfully to its final destination, so their elimination minimizes the consumption of network resources due to the continuity of their dispersion in the network.

ListeMAN $(\mathrm{AB})=\{$ ListeMAN $($ VAi $)\} \cup\{$ ListeMAN $($ VBi $)\}$

\subsubsection{Creating a List of Common Neighbors}

In this step each node compares the list of neighbors it receives with its own list to find the common neighbors. $\mathrm{VA}=\{\mathrm{VAi}=\mathrm{Ai}, \mathrm{i}=1 \ldots \mathrm{n}\}$

Where $\mathrm{n}$ is the number of neighbors of $\mathrm{A}$. $\mathrm{VB}=\{\mathrm{VBi}=\mathrm{Bi}, \mathrm{i}=1, \ldots, \mathrm{m}\}$

With m: number of neighbors of $B$

$\mathrm{VA} \cap \mathrm{VB}=\{\mathrm{Ni}, 1 \ldots \mathrm{n}\}$

$\mathrm{n}$ : number of neighbors shared between A and B.

$\checkmark \quad$ if the number of neighbors " $n$ " is even:

$$
\mathrm{VAB}=\{\mathrm{Ni} \in(\mathrm{VA} \cap \mathrm{VB}) 1 \ldots \mathrm{n} / 2\} .
$$

$\mathrm{VBA}=\{\mathrm{Ni}(\cap \mathrm{VA} V B)(2+\mathrm{n}) / 2\} \ldots \mathrm{n}$.

$\checkmark$ if the number of neighbors " $\mathrm{n}$ " is odd.

$$
\begin{aligned}
\mathrm{VAB} & =\{\mathrm{Ni} \in(\mathrm{VA} \cap \mathrm{VB}) 1 \ldots .(\mathrm{n}+1) / 2\} . \\
V B A & =\{N i(\cap V A V B),(n+3) / 2 \ldots n\} .
\end{aligned}
$$

\subsubsection{Updating the buffer}

After creating the list of messages that nodes should not accept in their future contacts and list of common neighbors (VA $\cap \mathrm{VB}$ ), the two nodes $\mathrm{A}$ and $\mathrm{B}$ function as follows: - They clean their buffers by eliminating unnecessary messages in the list ListeMAN (AB).

- Node A sends a list ListeMAN (AB) to its neighbors except those in the list VBA which will be supported by node $B$. - Node B sends a list ListeMAN (AB) to its neighbors except those in the list VAB that will be dealt with by node A. This implies that the neighbors of two nodes clean their buffers by eliminating unnecessary messages in the ListeMAN list $(\mathrm{AB})$. With this technique it is possible to increase the rate of messages to eliminate to release buffers of neighboring nodes and increase the probability that a message previously received by its destination stops circulating in the network.

\subsubsection{Transmission of messages \\ 6.1.4.1 Form of messages}

Table 1 Information contained in a message

\begin{tabular}{|l|l|l|l|l|}
\hline Message & $\mathrm{S}$ & $\mathrm{D}$ & $\left(\mathrm{hop}, \mathrm{t}_{\mathrm{i}}\right)=\left(\mathrm{N}_{1}, \mathrm{t}_{1}\right) \ldots\left(\mathrm{N}_{\mathrm{n}}, \mathrm{t}_{\mathrm{n}}\right)$ & size \\
\hline
\end{tabular}

Table 2. Information Contained in a Acknowledgement

\begin{tabular}{|l|l|l|l|l|l|}
\hline Ack & $D$ & $\mathrm{~S}$ & $\left(\mathrm{~K}_{\mathrm{i}}, \mathrm{t}_{\mathrm{i}}\right)=\left(\mathrm{k}_{1}, \mathrm{t}_{1}\right) \ldots\left(\mathrm{K}_{\mathrm{n}}, \mathrm{t}_{\mathrm{n}}\right)$ & $\left(\mathrm{S}, \mathrm{t}_{0}\right)\left\{\left(\mathrm{N}_{\mathrm{i}}, \mathrm{t}_{\mathrm{i}}\right)\right\}\left(\mathrm{D}, \mathrm{t}_{\mathrm{n}+1}\right)$ \\
\hline
\end{tabular}

S: Source, D: Destination, (hop, $t_{j}$ ) hop involved in transport of the message, ti: the time that the message resides in hop $\mathrm{N}_{\mathrm{i}-1}$ before being transmitted to $\mathrm{N}_{\mathrm{i}}$, Size: the size of the message. (Ki, ti): hop that participated in the transport of Ack message (acknowledgement messages).

$\left\{\left(\mathrm{S}, \mathrm{t}_{0}\right)\left\{\left(\mathrm{N}_{\mathrm{i}}, \mathrm{t}_{\mathrm{i}}\right)\right\}\left(\mathrm{D}, \mathrm{t}_{\mathrm{n}+1}\right)\right\}$ the path that the message takes to go from the source to the destination.

\subsubsection{Transmission of the Priority List.}

Each node classifies messages in its buffer; example node A searches for messages that have as destination node B, it classifies them in descending order based on their injection in the network and in ascending order based on their size and it is at this level that transmission starts.

- Method for creating the priority list: Node A consults the buffer messages before deciding: - If $\mathrm{B}$ is the source: it ignores the message.

- If $\mathrm{B}$ is the destination: it adds the message to the priority list. - If B belongs to the hops list that participated in the transport of the message to the moment of contact: it ignores the message.

Once the test is complete, the priority list is created before forwarding the messages in this list to node $\mathrm{B}$.

\subsubsection{Transmission or non-Transmission of Other Messages.}

In the case where the node B is not in the set of hops

$\{(\mathrm{S}, \mathrm{D},\{(\mathrm{Ni}, \mathrm{Ti})\}\}$ that is to say, it is neither a source nor a destination, nor the one of the nodes that participated in the transfer of messages, node A uses the information contained in the list of paths supplied by the set of paths extracted from the list ListeMAN (AB):

$\left\{\left(\mathrm{S}_{\mathrm{j}}, \mathrm{t}_{0}\right) \quad\left\{\left(\mathrm{N}_{\mathrm{i}}, \mathrm{t}_{\mathrm{i}}\right)\right\} \quad\left(\mathrm{D}_{\mathrm{j}}, \mathrm{t}_{\mathrm{n}+1}\right)\right\}$ : list of paths of messages successfully transmitted to their destination.

$\left\{\left(\mathrm{D}_{\mathrm{j}}, \mathrm{t}_{\mathrm{n}+1}\right)\left\{\left(\mathrm{K}_{\mathrm{i}}, \mathrm{t}_{\mathrm{i}}\right)\right\}\left(\mathrm{N}_{\mathrm{j}}, \mathrm{t}_{\mathrm{n}}\right)\right\}$ : list of acknowledgments paths. Node $A$, creates a list of pairs of nodes $\left(\mathrm{N}_{\mathrm{i}}, \mathrm{D}\right)$ called

Listech $(\mathrm{D}, \mathrm{Ni})=\{$ vector(frequency, hops average number $)\}$ which means that it extracts from all messages paths in list ListeMAN (AB), the number of times(frequency) when the two nodes are involved with delivering messages to their success destination and the average number of hops that separate in each path, then classifies messages in ascending order based on the number of occurrence of nodes $\mathrm{Ni}$ and $\mathrm{D}$ (frequency) and in descending order of hop number between them, thereafter, it selects from its neighbors which must carry the message. The nodes $\mathrm{N}_{\mathrm{i}}$ and $\mathrm{D}$ are very close if the number of hops between them is minimal, in this case there is a strong chance that the message reaches its destination without any problems.

\section{EXAMPLE}

Assume $\mathrm{A}, \mathrm{B}, \mathrm{C}$ and $\mathrm{F}$ are four intermittently connected mobile nodes and $\{\mathrm{B}, \mathrm{C}, \mathrm{F}\}$ are neighbors of $\mathrm{A}$, Table 3 and Table 5 represent the organization of messages at node A according to FIFO and Supp-Tran respectively, previous to the transmission and one of its neighbors now that $\{\mathrm{M} 7$, M11\} are within reach to their destination. It is assumed that each message transmission time is 1 second while total DTN transmission time is 4 seconds.

\section{Case 01: Forwarding Sequence with FIFO strategy}

Table 3 represents the array of messages at node A before the transmission. With FIFO strategy, node A transmits the messages:

$\{(\mathrm{M} 1, \mathrm{D}),(\mathrm{M} 2, \mathrm{~B}),(\mathrm{M} 3, \mathrm{C}),(\mathrm{M} 4, \mathrm{~B})\}$ to Node B.

$\{(\mathrm{M} 5, \mathrm{D}),(\mathrm{M} 6, \mathrm{~F}),(\mathrm{M} 7, \mathrm{D}),(\mathrm{M} 8, \mathrm{~F})\}$ to Node C.

$\{(\mathrm{M} 9, \mathrm{C}),(\mathrm{M} 10, \mathrm{~B}),(\mathrm{M} 11, \mathrm{D})\}$ to Node F. 
Table 3. Snapshot of node messages W.R.T FIFO

\begin{tabular}{|c|c|c|}
\hline Msg & Destination & Arrival Time \\
\hline M1 & D & 127 \\
\hline M2 & B & 123 \\
\hline M3 & C & 120 \\
\hline M4 & B & 124 \\
\hline M5 & D & 122 \\
\hline M6 & F & 34 \\
\hline M7 & D & 30 \\
\hline M8 & F & 20 \\
\hline M9 & C & 14 \\
\hline M10 & B & 11 \\
\hline M11 & D & 9 \\
\hline
\end{tabular}

TABLE 4 . «FIFO » After Transmission

\begin{tabular}{|c|c|c|}
\hline Node & Msg & Destination \\
\hline \multirow{4}{*}{ A-------->B } & $M 1$ & D \\
\cline { 2 - 3 } & $M 2$ & B \\
\cline { 2 - 3 } & $M 3$ & $C$ \\
\cline { 2 - 3 } & $M 4$ & $B$ \\
\hline \multirow{4}{*}{ A-------->F } & $M 5$ & $D$ \\
\cline { 2 - 3 } & $M 6$ & $F$ \\
\cline { 2 - 3 } & $M 7$ & $D$ \\
\cline { 2 - 3 } & $M 8$ & $F$ \\
\hline \multirow{4}{*}{ A-------->C } & $M 9$ & $C$ \\
\cline { 2 - 3 } & $M 10$ & $B$ \\
\cline { 2 - 3 } & $M 11$ & $D$ \\
\hline
\end{tabular}

Table 4 illustrates the result after the transmission involving A and its neighbors using FIFO under epidemic routing protocol. The result shows that with FIFO strategy messages M3 and M10 will be forwarded to other nodes relay, while the good destination of each message appears among its neighbors and M1, M5 continue to travel although one of its copies has already arrived to its destination which can cause the consumption of the network resources.

Case 02: Forwarding sequence with "Supp-Tran" strategy Table 5 depicts the organization of messages at node A by Supp-Tran previous to transmission.
Table 5. Snapshot of node messages W.R.T Supp-Tran

\begin{tabular}{|c|c|c|}
\hline Message & Destination & Arrival Time \\
\hline M2 & B & 123 \\
\hline M4 & B & 124 \\
\hline M10 & B & 11 \\
\hline M6 & F & 34 \\
\hline M8 & F & 20 \\
\hline M3 & C & 120 \\
\hline M9 & C & 143 \\
\hline M1 & D & 127 \\
\hline M5 & D & 122 \\
\hline M7 & D & 30 \\
\hline M11 & D & 98 \\
\hline
\end{tabular}

With this strategy, while being based on the list of the messages that have already arrived to their destinations, the node A deletes the messages $\{M 7, M 11\}$ from its buffer then it sends the messages $\{(\mathrm{M} 2, \mathrm{~B}),(\mathrm{M} 4, \mathrm{~B}),(\mathrm{M} 10, \mathrm{~B})\},\{(\mathrm{M} 6, \mathrm{~F})$, $(\mathrm{M} 8, \mathrm{~F})\}$ and $\{(\mathrm{M} 3, \mathrm{C}),(\mathrm{M} 9, \mathrm{C})\}$ respectively towards the good destinations $\mathrm{B}, \mathrm{H}$ and $\mathrm{C}$ which results in a rise of delivery ratio. Regarding the messages whose destination does not appear among the neighbors, Node A consults the list Listech $\left(\mathrm{D}, \mathrm{N}_{\mathrm{i}}\right)$ [Table 6] to choose the node that has more chances to transport them.

Table 6. LISTECH(D,Ni) of node a Created By "Supp-Tran" in Section 6.

\begin{tabular}{|c|c|c|}
\hline pair of nodes & frequency & Average hops \\
\hline D:C & 11,7 & 0 \\
\hline D:B & 5,87 & 3 \\
\hline D:H & 3 & 3 \\
\hline D:F & 3 & 5 \\
\hline D:E & 2 & 7 \\
\hline D:A & 1 & 1 \\
\hline
\end{tabular}

Indeed, for the two messages $\{(\mathrm{M} 1, \mathrm{D}),(\mathrm{M} 5, \mathrm{D})\}$ intended for the Node D, the node A will certainly choose the node C which has the best value of (frequency, hops average number) compared to the other nodes. Table 7 displays the result after the transmission of messages by "Supp-Tran".

\section{SIMULATION AND RESULTS}

\subsection{Simulation Environments}

This section presents the results of the comparison between the forwarding strategies FIFO and Sup-Tran presented in Section 6, regarding their effectiveness under epidemic routing protocols. All simulations are done with the ONE (The Opportunistic Network Environment Simulator) [1] 
Table 7. «Supp-Tran » after Transmission

\begin{tabular}{|c|c|c|}
\hline Node & Msg & Destination \\
\hline \multirow{3}{*}{$A------->B$} & M2 & \multirow{3}{*}{$\mathrm{B}$} \\
\hline & M4 & \\
\hline & M10 & \\
\hline \multirow{2}{*}{ A--------->F } & M6 & \multirow{2}{*}{$\mathrm{F}$} \\
\hline & M8 & \\
\hline \multirow{4}{*}{ A------->C } & M3 & \multirow{3}{*}{$\mathrm{C}$} \\
\hline & M9 & \\
\hline & M1 & \\
\hline & M5 & \\
\hline & M7 & \multirow{2}{*}{ deleted } \\
\hline & M11 & \\
\hline
\end{tabular}

written in Java to evaluate the performance of the two methods. The ONE was created by Helsinki University and provided the map of the Helsinki area.

In the simulation, there are two different nodes that may generate and receive a message. One is a pedestrian and the other is a vehicle. The number of two different nodes is 40 and 6 respectively for pedestrian and vehicle. These two types of nodes are moving with different mobility along the map of Helsinki area.

For the pedestrian, the walking speed (i.e., $0.5 \mathrm{~m} / \mathrm{s} \sim 1.5 \mathrm{~m} / \mathrm{s}$ ) is applied. The moderate speed (i.e., $6 \mathrm{~m} / \mathrm{s} \sim 12 \mathrm{~m} / \mathrm{s}$ ) is applied to the vehicle. The details of the simulation parameters are shown in Table 8.

\subsection{Performance Analysis}

To evaluate the proposed method compared with FIFO under epidemic routing protocol, the simulation has to consider performance metrics such as delivery rate, communication overhead, and number of hops. It is evident that the higher delivery rate means better performance on successful data delivery. However, the effort to get the higher delivery rate, a routing protocol has to send the more data into the networks, these additional data packets may result in communication overhead. Therefore, both delivery rate and communication overhead are analyzed simultaneously.

Finally, average number of hops is compared to demonstrate consumption of resources. clearly, DTNs accept a tolerable delay for message delivery. On the other hand, some applications do not accept higher number of hops to deliver messages from the source to the destination.

\subsection{Simulation Results}

\subsubsection{By Varying Time Intervals}

Fig 2 shows that increasing the simulation time from $10 \mathrm{~K}$ to $80 \mathrm{~K}$ increases the probability of deliverance for both method, which can be explained as more nodes are traveling for a long time, the more they encounter another nodes so they may exchange more messages, but for each time interval, the results show that the strategy Supp-Tran has better performance than the FIFO strategy in terms of messages that can be delivered to their destination.

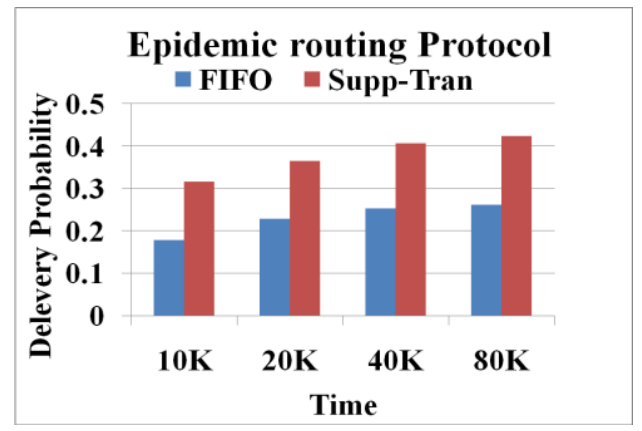

Fig 2 . Delivery Probability w.r.t Time

Fig 3 shows the results of the evaluation in terms of the overhead ratio of FIFO and Supp-Tran by varying the time interval from $10 \mathrm{~K}-80 \mathrm{~K}$ under epidemic routing protocol. As shown, the overhead generated by the proposed method is lower than the FIFO strategy in all interval time, which means that with Supp-Tran strategy, the number of messages that continue traveling in the network without reaching their destination. Unlike, with FIFO strategy where this number is higher which means that the messages moving around the network for a long time which causes that the messages consume the network resource.

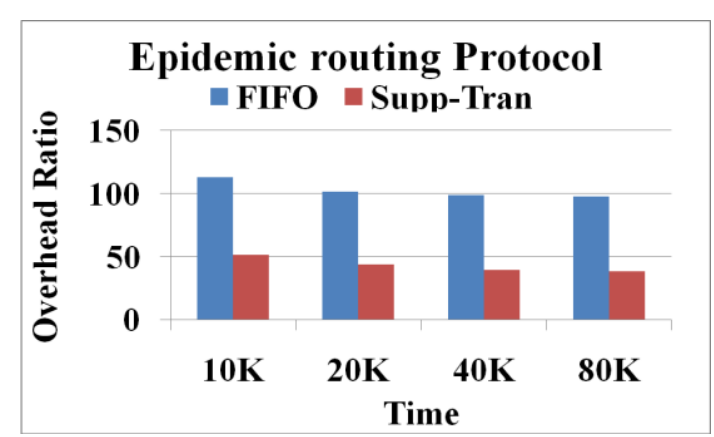

Figure 3. Overhead w.r.t Time

Fig 4 explains the average hops number of the proposed strategy compared to FIFO technique, as the result show, the proposed approach has a smaller pattern of the average hops number. It can see clearly that at various time intervals the hop-count average of "Supp-Tran" is lower than FIFO. The proposed method uses a number of hops slightly greater than the strategy FIFO which is evident because the Supp-Tran method tries to choose the best hops having a higher probability or greater chance to deliver messages, unlike the FIFO technique that transmits the messages based on their arrival time without checking if the relay nodes will encounter destination or not which causes that some nodes carry the messages even if it will never meet the destination, even if it has a small number of hops, it does not mean that is better because the delivery probability is low as its shown above in Fig 2. 


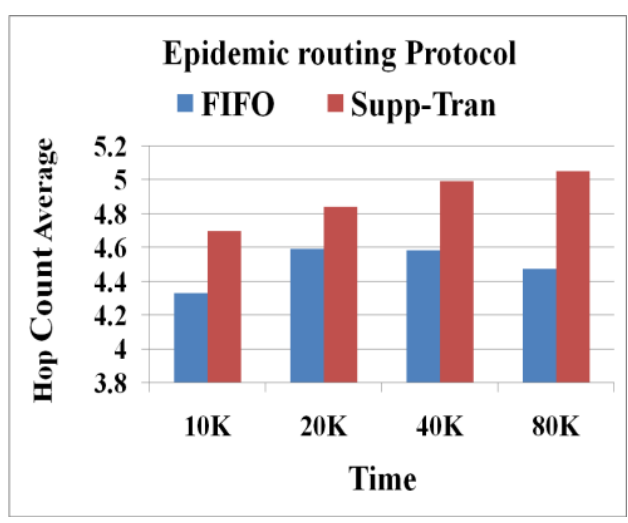

Fig 4. Hop count average w.r.t Time

\subsubsection{By Varying Transmission range}

Fig 5 compares FIFO and Supp-Tran strategy in terms of delivery probability by increasing the transmission range. The Supp-Tran strategy proves better delivery probability than FIFO, since increasing the transmission range increases the number of neighbors, which allows nodes using Supp-Tran to deliver the message as close as possible to their destination by choosing the most appropriate hop.

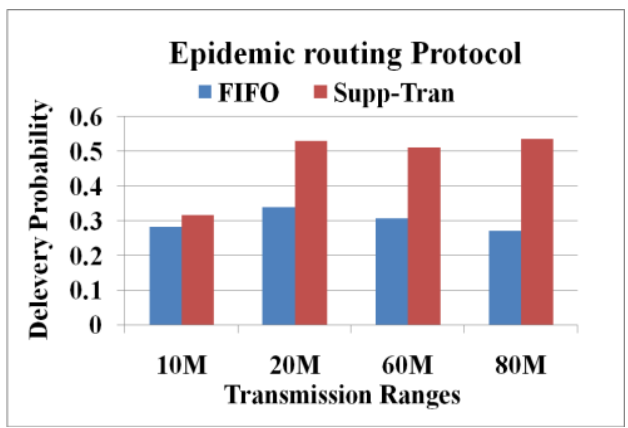

Figure 5. Delivery probability w.r.t Transmission range

Fig 6 shows that in all transmission ranges the overhead of "Supp-Tran" is lower than FIFO strategies. This means that the strategy Supp-Tran is able to choose the best relay to transport messages to the right destination that is reflected in the low value of overhead.

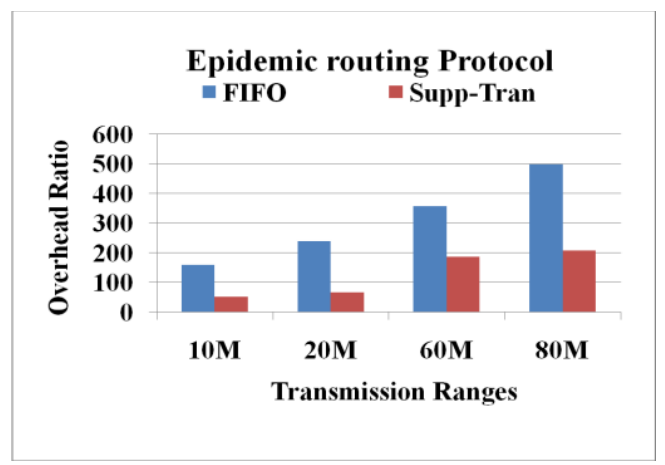

Figure 6 Overhead w.r.t Transmission range
Fig 7 presents the impact of transmission range on hop count. It shows that the hops count with FIFO strategy is lower than Supp-Tran. The sup-Tran, especially in the delivery probability and overhead ratio, is more efficient than FIFO.

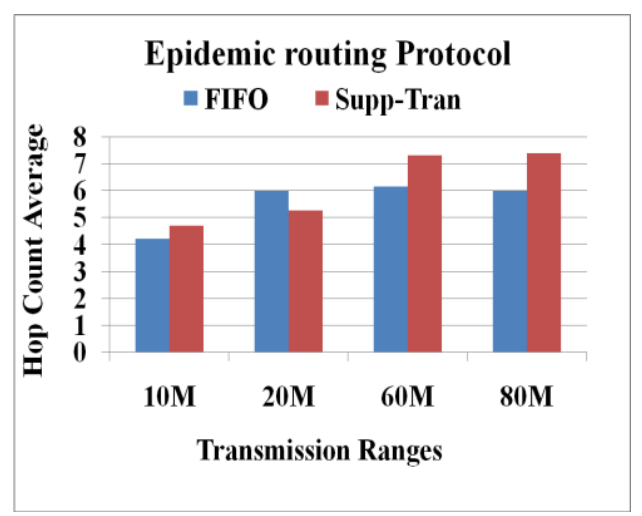

Figure 7 hop count Average w.r.t Transmission range.

\section{CONCLUSIONS}

This article proposes a strategy called " Supp -Tran ", which aims to optimize routing in delay tolerant networks to use the network resources in an efficient manner contrary to the FIFO technique, as its name indicates, the proposed technique consists of two phases, the 'Supp' which means that the encountered nodes start to clean their buffer before deciding to transmit messages that are in their buffer. They exchange the list of messages that are successfully delivered to delete the remaining copies that still circulate in the network to release their buffers as well as those of their neighbors. The phase 'Tran' starts with the transmission of the remaining messages in their buffer based on the list of neighbors and the list formed by the paths of messages delivered to calculate the frequency of meeting between destination and neighboring nodes of the two nodes that are in communication as well as the number of hops between them. During the phase "Supp", a proposed strategy optimizes routing epidemic by increasing the rate of the removed copies of messages that have been delivered to their destination. Indeed, the list formed by considering the list of the deleted messages of the encountered nodes and their neighbors will be forwarded to the neighbors to clean their buffer. During the " Tran" Phase, the proposed technique optimizes routing epidemic in terms of selecting relay nodes that will be carrying the messages. In fact, it uses the paths contained in the deleting messages' list to calculate the number of times the relay nodes have met the final destination of messages. This technique enables the reduction of the overhead and increases the delivery probability of messages compared to FIFO strategy. 


\section{REFERENCES}

[1] V. Cerf and al. 2001. Interplanetary internet (IPN): Architectural definition.

[2] J. Crowcroft, E. Yoneki, P. Hui, and T. Henderson. 2008.Promoting tolerance for delay tolerant network research. SIGCOMM.

[3] A. Vahdat and D. Becker. 2000. Epidemic routing for partially-connected ad hoc networks, Tech. Rep.

[4] Spyropoulos, K. Psounis and C. S.Raghavendra. 2005. Spray and wait: an efficient routing scheme for intermittently connected.
[5] Ari Keränen, Jörg Ott and Teemu Kärkkäinen. 2009. The ONE Simulator for DTN Protocol Evaluation.

[6] T. Spyropoulos, K. Psounis, and C. S. Raghavendra. 2005. Spray and wait: Efficient routing in intermittently connected mobile networks," in Proc.ACM SIGCOMM Workshop on Delay Tolerant Networking (WDTN).2005

[7] A. Lindgren and A. Doria .2012.Probabilistic Routing Protocol for Intermittently Connected Networks.

[8] Ashraf, Al-Fagih, Hossam and S.Hassanein.2012. Routing Schemes for Delay-Tolerant Networks: An Applications Perspective Technical Report. 